\title{
Competencias para la traducción jurídica: modelos, enfoques y percepción del profesorado
}

\author{
Competences for legal translation: models, approaches, \\ and perception of lecturers
}

\author{
Robert Martínez-Carrasco
}

Universitat Jaume I. rcarrasc@uji.es

Recibido: 30.04.2019. Aceptado: 07.08.2019

Resumen: El artículo que presentamos analiza los elementos diferenciadores de la educación por competencias en traducción jurídica y las exigencias metodológicas en el aula. Mediante una metodología de obtención de datos de carácter cualitativo y cuantitativo, el artículo pretende sondear la realidad en el aula de traducción jurídica en España a través del análisis exhaustivo de las guías docentes del conjunto de módulos ofertados en traducción jurídica. Los resultados, contrastados con una serie de entrevistas semiestructuradas a docentes de traducción jurídica en activo, contextualizan la práctica docente y dan voz al profesorado universitario, permitiéndole compartir sus percepciones, puntos de vista y experiencia en el aula de traducción.

Palabras clave: traducción jurídica; educación por competencias; competencia traductora; didáctica de la traducción; metodología docente.

\begin{abstract}
The article we present below analyses the characteristic elements of a competence-based approach in legal translation education, together with the methodological challenges it poses for translation students. Using both qualitative and quantitative research methods, the article aims to provide an accurate depiction of the way legal translation is taught in Spain through the analysis of all existing legal translation syllabi at undergraduate level. The results were contrasted with a number of semi-structured interviews to legal translation lecturers, allowing us to explore what happens in the legal translation classroom and reflect, at the same time, on the lecturers' perceptions, experiences and opinions about it.
\end{abstract}

Keywords: legal translation; competence-based teaching approach; translation competence; translation education; teaching methodology.

》 Martínez-Carrasco, Robert. 2019. “Competencias para la traducción jurídica: modelos, enfoques y percepción del profesorado”. Quaderns de Filologia: Estudis Lingüistics XXIV: 267-290. doi: 10.7203/QF.24.16311 



\section{Introducción}

El desarrollo exponencial y la convergencia de sectores altamente tecnológicos como la robótica, la nanotecnología, la inteligencia artificial o las tecnologías de la información y de la comunicación han llevado a muchos a plantearse la inminencia de una cuarta revolución industrial basada en la automatización de procesos (World Economic Forum, 2017). En ese contexto, la traducción, en tanto que elemento indispensable en la globalización de productos y servicios, cumple un papel instrumental muy necesario que, dado el ingente volumen de palabras ${ }^{1}$ y la inmediatez con la que se llevan a cabo transacciones económicas e intercambios de todo tipo, requerirá un cambio de paradigma significativo en la industria de la traducción caracterizado no ya sólo por los tiempos de trabajo y volúmenes de palabras, sino también por las lenguas protagonistas del proceso, determinadas por los agentes económicos, políticos y sociales hegemónicos del momento (Lommel \& DePalma, 2017).

En el caso particular de la traducción jurídica, Borja Albi y Martínez-Carrasco (2019: 197) apuntan a la localización y desarrollo de contenido jurídico multilingüe como uno de los campos con mayor proyección laboral dadas las previsiones de crecimiento y expansión de la industria de la traducción jurídica. Así, pese a que los procesos de automatización y desarrollo de motores de traducción automática basados en redes neuronales afectará a los tiempos y modos de trabajo, la traducción jurídica, dado el nivel de anisomorfismo que presentan los diferentes ordenamientos jurídicos implicados, necesitará de profesionales altamente especializados capaces de discernir aquellas asimetrías que resultan de la naturaleza de los textos jurídicos y que suponen uno de los elementos más característicos de este tipo de traducción (Borja Albi, 2013: 34).

Fruto de esta reflexión sobre lo que parece ser el futuro de la profesión del traductor jurídico, cabría plantearse si los planes de estudio actuales están preparados para dar respuesta a las necesidades cambiantes y estrechamente relacionadas con la tecnología que parecen avecinarse en la industria de la traducción. En última instancia, además, subyace

\footnotetext{
${ }^{1}$ Se calcula que actualmente se traducen cien mil millones de palabras al día mediante sistemas de traducción automática (Casacuberta Nolla \& Peris Abril, 2017: 67).
} 
el concepto mismo de competencia traductora, aquellos conocimientos declarativos, procedimentales y actitudinales que los docentes de traducción jurídica consideran necesarios en el proceso de socialización progresiva de sus alumnos.

Bajo este prisma, a la par que los futuros traductores desarrollan y adquieren mayor competencia en un ámbito socio-profesional concreto -en este caso, el de la traducción jurídica- mayor es su grado de implicación directa en la comunidad socio-profesional a la que aspiran unirse, incrementando considerablemente su participación periférica legítima (Lave \& Wenger, 1991). Por ende, el aprendizaje de la traducción jurídica, y el desarrollo de la competencia traductora en traducción jurídica, se convierte en una actividad situada que se ve favorecida enormemente por entornos de aprendizaje (quasi) profesionales donde el alumno puede hacer suyos comportamientos social y profesionalmente consensuados por la comunidad de práctica en cuestión al tiempo que contribuye a la misma a través de su participación periférica. Así, al entender el aprendizaje de la traducción como un proceso progresivo intrínseco al ejercicio profesional, se vincula el desarrollo de la competencia traductora a uno de los pilares fundamentales del EEES: el aprendizaje a lo largo de la vida (Marco, 2016: 40).

El enfoque por competencias, hoy completamente asentado en la educación de tercer ciclo en nuestro país, encuentra precisamente en el EEES la justificación y legitimación institucional que necesita y que ha sido, en parte, responsable del triunfo del paradigma de las competencias en educación terciaria (Martínez-Carrasco, 2017a: 163-164). Sin ir más lejos, el mismo Comunicado de Ereván (2015) resume perfectamente el cambio de paradigma educativo que se ha vivido en España con la consolidación del EEES. Por un lado, abogando por el factor empleabilidad como elemento imprescindible en educación superior, factor que realza la doble vertiente declarativa y procedimental del enfoque por competencias ("asegurar que los estudiantes adquieran las competencias relevantes de cara a su empleabilidad (...) compaginando el contenido teórico y práctico en las asignaturas"); por otro, promoviendo "entornos de aprendizaje centrados en el estudiante que exploren los beneficios de las tecnologías digitales".

Sin embargo, para que tales reformas de carácter pedagógico y metodológico tengan calado, se necesita la complicidad de aquellos docentes encargados de dar forma a la educación superior en España. Si bien 
es cierto que el Comunicado de París (2018), fruto de la última cumbre europea en materia de educación superior, hace referencia explícita a la necesidad de dotar al EEES de "iniciativas institucionales, nacionales y europeas encaminadas a mejorar la preparación docente de los profesores universitarios, su formación pedagógica continuada y el reconocimiento de aquellas prácticas docentes innovativas y de calidad" (ibid.: 4), lo cierto es que, en general, los docentes universitarios suelen carecer de una base pedagógica firme y se asume que aquellos que son expertos de una materia en concreto saben cómo enfrentarse a un aula universitaria (Kelly, 2008: 102).

Sea como fuere, los estudios de traducción cuentan actualmente con numerosos estudios empíricos que refuerzan el paradigma de las competencias en la didáctica de la traducción hasta el punto que se hace difícil hablar de enseñanza de la traducción jurídica sin recurrir al constructo competencia. Sí se hace necesario, sin embargo, desgranar cuáles son las exigencias metodológicas en el aula de traducción jurídica que se deducen de los modelos de competencia traductora planteados hasta la fecha, en particular aquellos planteados específicamente para la traducción jurídica, recogidos, entre otros, por Martínez-Carrasco (2017b: 262-279) o Soriano-Barabino (2018: 221-226).

\section{El paradigma de las competencias en la didáctica de la traducción}

El Documento Marco para la integración del Sistema Universitario Español en el EEES (2003), junto con el Real decreto RD1393/2007, ponen de manifiesto la voluntad de diseñar un sistema universitario basado en la educación por competencias, y llama a las universidades españolas a adaptar, en el proceso de creación y consolidación de los nuevos grados universitarios, modelos de enseñanza y aprendizaje acordes con el trabajo por competencias. En el caso particular de la traducción este cambio no supone un cambio drástico respecto a la manera de articular las antiguas licenciaturas en traducción. Al fin y al cabo, como disciplina, la traducción contaba con dos ventajas competitivas en el proceso de adopción del paradigma de las competencias. Por una parte, la naturaleza preeminentemente práctica de los estudios de traducción casaba con la dimensión procedimental del trabajo por competencias, utilizado principalmente, hasta ese momento, por los programas de 
formación profesional, más orientados al mercado laboral (Halász \& Michel, 2011). Por otra, la naturaleza más joven de los estudios de traducción dotaba a los planes de estudio y departamentos de traducción de mayor flexibilidad para adoptar los cambios pertinentes.

En el área de la traducción puede afirmarse que dos obras marcan un antes y después en la enseñanza de la traducción por competencias tal y como la conocemos actualmente: Developing Translation Competence, de Schäffner y Adab (2000) y, en el mismo año, el giro constructivista que propone Kiraly en A social constructivist approach to translator education (2000). Ambas publicaciones suponen el inicio de un debate, el de la competencia traductora, a la que se ha referido con calificativos diferentes a lo largo de los años: translation skill (Lowe, 1987: 57), translation ability (Lowe, 1987: 57; Stansfield, Scott, \& Kenyon, 1992), translation performance (Wilss, 1989: 129), transfer competence (Nord, 1991: 161), translational competence (Pym, 1991; Toury, 1995: 250-51; Chesterman, 1997: 147; Hansen, 1997: 205; Li, 2001), translation competence (Kelly, 2002; Neubert, 2000; PACTE, 2003, entre otros), etc. Incluso se ha subrayado la diferencia entre translation competence y translator competence, esta última referida no ya sólo a la habilidad interlingüística de traducir sino también a la movilización de elementos socio-profesionales que hace de un traductor un miembro de su comunidad de práctica.

En cualquier caso, todas las definiciones de competencia traductora hasta la fecha admiten que se trata de un constructo situado, dependiente del contexto en que se moviliza (Edwards-Schachter et alii, 2015: 24), de vocación práctica, relacionada con un saber hacer procedimental (Lasnier, 2001: 30) y resultado de la combinación de elementos cognitivos (conocimientos, habilidades), afectivos (motivación, personalidad, actitudes), psicomotores y conductuales (hábitos) y psicofisiológicos (memoria oral y visual, por ejemplo), elementos que se resumen, tal y como apunta Martínez-Carrasco (2017b: 154-155) en forma de:

(...) a polyhedral, complex construct composed of a dynamic cluster of forming elements that apply to specific, situated work conditions. Knowledge, skills, behaviour, and attitudes, whose weight in the overall competence construct may vary according to the particular task to be performed, play the most relevant role in the said cluster. 
Al entender la noción de competencia en traducción como un sistema complejo, dinámico, los límites del constructo se expanden, aceptando que la competencia traductora es mutable, cambiante, en función de dos parámetros: la interacción con el medio y la situación en que se movili$\mathrm{za}$, y las propiedades y características emergentes fruto de la interacción y movilización interna de los distintos elementos que la componen (De Bot et alii, 2007: 8).

Este enfoque, tomado de la Teoría de los Sistemas Dinámicos (TSD), entiende que para que un sistema se desarrolle (la sub-competencia lingüística en los modelos de competencia traductora, por ejemplo) se necesita un aporte mínimo de energía y recursos proveniente del flujo general que mantiene el sistema y permite la emergencia de estructuras cognitivas. La TSD aplicada a la competencia traductora, en línea con los estudios de Göpferich (2013), por ejemplo, o el modelo de competencia traductora de Kiraly (2016a: 71) "explicaría por qué cada una de las sub-competencias dentro del esquema general se desarrollan a ritmos distintos en el proceso de aprendizaje del los alumnos, por qué no siempre evolucionan de forma lineal, o por qué ciertas competencias o grupos de competencias sirven como percusores para el desarrollo de otras y tienen un efecto directo en su desarrollo" (Martínez-Carrasco, 2017a: 168).

\subsection{La competencia en traducción jurídica}

Cao (2014: 107), a propósito de la enseñanza de la traducción jurídica, afirma que aunque un buen traductor jurídico tiene que ser, antes que nada, un traductor generalista competente, no tiene por qué darse el caso al revés. Ciertamente, los rasgos característicos de la traducción jurídica, sea la misma naturaleza del derecho, el lenguaje jurídico, las diferencias sistémicas entre familias del derecho, etc., apuntan a que los modelos de competencia en traducción general pueden llegar a ser reduccionistas cuando se trata de vehicular la enseñanza de la traducción jurídica, ya que no tienen en cuenta las particularidades de esta modalidad de traducción. Así, en textos altamente especializados, el componente temático adquiere una importancia principal que condiciona no ya la comprensión del texto, sino poder acceder a las diferentes formas discursivas de la comunidad en que esos textos aparecen. Ordóñez López (2015: 113-114), en ese sentido, se refiere a la necesidad de que los 
alumnos de traducción jurídica conozcan no ya sólo la terminología específica del campo, sino también principios de razonamiento jurídico, capacidad de análisis de los textos jurídicos y conocimiento profundo de los ordenamientos jurídicos implicados, entre otros. Siempre subrayando el factor conceptual y el peso del derecho en traducción jurídica, se han presentado diferentes propuestas de modelos de competencia traductora específicos para este tipo de traducción, que pasamos a revisar sucintamente a continuación.

El primer modelo de competencia traductora exclusivamente diseñado para la traducción jurídica no lo encontramos hasta 2011, de la mano de Prieto Ramos, en el seno de la red EMT (Prieto Ramos, 2011). Previamente, Šarčević (1997: 113-114) o Cao (2007: 41) proporcionan un listado de habilidades y conocimientos que el traductor jurídico debe poseer, a su juicio, frente al traductor generalista (terminología jurídica, razonamiento lógico, razonamiento jurídico, derecho comparado, etc.), pero sus propuestas no están articuladas a través de un modelo de competencia concreto. Cabe mencionar que en ambos casos no se hace referencia alguna a los conocimientos lingüísticos, por no ser exclusivamente característicos de la traducción jurídica sino un pre-requisito para la traducción, independientemente de la modalidad. Más adelante, Cao (2014) retoma esas reflexiones previas y, utilizando como base el modelo de competencia que propone el EMT (2009), la autora propone un modelo de competencia traductora basado en cuatro variables "interacting in a legal situational context" (ibid.: 112-113).

El modelo de Prieto Ramos (ibid:: 11-12), de carácter multicompetencial y basado en premisas declarativas y operativas, parte de aportaciones previas en competencia para la traducción general (PACTE, Kelly, etc.) e integra los elementos característicos de la traducción jurídica mencionados arriba en un constructo orientado a la traducción como proceso donde la competencia estratégica coordina el conjunto de la competencia traductora (ibid.: 18). Particularmente eficaz en lo que se refiere a la sistematización de procesos en traducción jurídica relacionados con la identificación de problemas de traducción, categorización de problemas y aplicación de patrones regulares en la solución de problemas de traducción, el modelo de Prieto Ramos recurre a los principios prácticos del derecho comparado (análisis contrastivo de conceptos en ordenamientos jurídicos diferentes, por ejemplo), y a la 
práctica profesional de la traducción jurídica, para dar forma al modelo de competencia que presenta.

Por su parte, Piecychna (2013: 155) propone un modelo basado en la hermenéutica que se aleja sensiblemente del resto de propuestas de competencia traductora y se centra en las habilidades que necesita el traductor en el momento en que se enfrenta a su texto. Su modelo, competencial y dispuesto en círculos concéntricos, otorga especial relevancia a la sub-competencia psicológica, que ocupa el círculo central en el modelo:

The "self-reflection upon one's own skills and knowledge; reflection upon one's own cultural and social position as a legal translator; acceptance of one's own limitations and possible lack of skills or knowledge; acceptance of the subjective nature of the translational process; self-criticism; self-motivation; willingness to develop one's own knowledge; willingness to pursue a career as a legal translator; attitude towards translation work; being a responsible, curious, patient, creative, hard-working, diligent, methodical, devoted, and imaginative person; the ability to identify and solve problems with appropriate strategies and techniques; the ability to analyse and interpret texts".

Quizá el aspecto más controvertido del modelo, criticado duramente por Scarpa y Orlando (2017: 25) es el hecho de que, en su modelo, Piecychna habla del traductor jurídico como un lingüista con especialización en traducción jurídica o un jurista experto en lengua con capacidad de interpretar jurídicamente un texto. Tal afirmación, en palabras de Scarpa y Orlando, entra en contradicción directa con la formación a medida que propone Prieto para los traductores jurídicos (2011: 19) o la afirmación de Šarčević (1997: 91) de que el traductor jurídico debe abstenerse de interpretar el texto en el sentido jurídico de la palabra.

Kordić (2016), en el contexto croata, y en concreto en el del desarrollo de programas de formación continua para traductores jurídicos, propone un modelo multicompetencial que recurre, al igual que Piecychna arriba, a la hermenéutica y a la capacidad de interpretar textos jurídicos por parte del traductor. Además, el modelo utiliza las expresiones "traductor jurídico" y "jurista-lingüista" de forma intercambiable, lo que, de nuevo, vuelve a desdibujar la frontera entre dos profesiones que, pese a ser similares hasta cierto punto, difieren en un aspecto vital que marca 
las necesidades principales en los programas de formación y enseñanza específicos para traductores jurídicos: el conocimiento del derecho.

Por último, el modelo de competencia en traducción jurídica más reciente hasta la fecha es el propuesto por Scarpa y Orlando (2017), en el seno del programa QUALETRA (JUST/2011/JPEN/AG/2975), en relación con la directiva europea 2010/64/EU relativa al derecho a interpretación y traducción en los procesos penales. Antes de presentar el modelo de competencia en cuestión, cuya finalidad es la de crear un marco formativo de cara a la acreditación de traductores jurídicos altamente especializados en procesos penales, los autores hacen un repaso de diferentes proyectos y estudios que han tenido un alcance similar, tales como el proyecto Aequitas: Access to Justice across Language and Culture in the EU (98/GR/13) (Hertog, 2003) y el Building Mutual Trust Project (JLS /2007/219). Respecto al primero de ellos, los autores subrayan el hecho de que el modelo de competencia que presenta Aequitas no parece estar fundamentado en la traducción jurídica sino ser un modelo de aplicación general cuya única marca diferenciadora sería el conocimiento de los sistemas civil y penal de los ordenamientos jurídicos correspondientes. El segundo de ellos, Building Mutual Trust, presenta, según los autores, las competencias que propone Aequitas junto con aquellas identificadas en el Final Report of the Reflection Forum on Multilingualism and Interpreter Training (Hertog, 2009).

Partiendo de esa base, y tomando como modelo el modelo de competencia traductora jurídica que propone Prieto arriba, Scarpa y Orlando (ibid.: 21-36) presentan un modelo extenso, detallado, y con énfasis en la dimensión profesional, de las competencias del traductor jurídico en el contexto europeo. El modelo en cuestión está formado por los siguientes elementos: competencia de prestación de servicios de traducción, competencia lingüística, competencia intercultural, competencia de documentación, competencia temática y competencia tecnológica. Para cada una de ellas, el modelo de QUALETRA desgrana diferentes habilidades y conocimientos propios de la traducción jurídica.

\section{Metodología y método en la didáctica de la traducción jurídica}

Llegados a este punto, y de cara a tratar cuestiones prácticas relacionadas con el desarrollo de la competencia traductora jurídica en el aula, convendría hacer una diferenciación entre tres elementos clave que a 
menudo causan cierta confusión entre los docentes legos en didáctica: el modelo pedagógico, el método didáctico y la metodología docente.

Por método entendemos la manera que tiene un docente de poner en marcha su modelo pedagógico, integrando determinados principios, prácticas y formas de evaluación en virtud de su propio estilo educativo. El EEES, por ejemplo, apuesta por un modelo pedagógico centrado en el alumno y una visión posmoderna de la cognición y del aprendizaje, cosa que conlleva toda una serie de exigencias metodológicas, es decir, el conjunto de técnicas, estrategias y recursos que permiten al docente concretar su método didáctico en un contexto determinado.

En el caso de la didáctica de la traducción jurídica, hemos visto como el fin último parece ser promover que los egresados se comporten y actúen como profesionales de su ámbito, facilitando su socialización a través de, entre otros, actividades, tareas y proyectos de traducción (González-Davies \& Enríquez-Raído, 2016: 1). Dos modelos pedagógicos apuntalan tal creencia docente: el aprendizaje significativo ( $\mathrm{Au}-$ subel, 1960), por el que los alumnos generan estructuras cognoscitivas nuevas en función de aquellas que poseían previamente, y el aprendizaje situado que, tal y como apuntan González-Davies y Enríquez-Raído (ibid.) otorga un papel fundamental al contexto y los elementos contextuales (factores institucionales, sociales y geográficos, creencias y costumbres autóctonas, etc.).

La manera de trabajar esta socialización situada de los alumnos, en aras de un aprendizaje predominantemente significativo, estaría pues relacionada con el trabajo colaborativo y el uso de materiales reales en un encargo que represente, en la medida de lo posible, entornos de trabajo reales. La literatura acerca de la metodología en la didáctica de la traducción jurídica recoge ciertamente las reflexiones de corte epistemológico que formulamos aquí, y, así, no es infrecuente encontrar trabajos donde se ensalza el trabajo por proyectos y tareas en el aula (Borja Albi, 2007; Andújar Moreno \& Cañada Pujols, 2011; Galán Mañas, 2011; Macías Otón, 2016) en tanto que "multi-competence assignments" (González-Davies \& Scott-Tennet, 2005: 17).

Algo similar ocurre con el uso de materiales auténticos en el aula, que, en palabras de Galán Mañas (ibid.: 110) pueden ayudar a desarrollar competencias tanto específicas de la traducción jurídica como competencias genéricas del currículum relacionadas, por ejemplo, con la inclusión de elementos socio-profesionales en el aula (ya que se trata de 
un encargo con materiales auténticos). El uso de materiales auténticos permitiría trabajar cuestiones relacionadas con la facturación, por ejemplo, las tarifas de mercado, relación y trato con el cliente, etc. (Biel, 2011: 72).

El trabajo colaborativo es otro de los elementos que aparece en la literatura sobre didáctica de la traducción, quizá en sintonía con el enfoque constructivista vigotskiano al que nos hemos referido arriba. Macías (ibid.: 140-145), por ejemplo, da cuenta de una experiencia docente en el aula de traducción jurídica encaminada, en palabras de la autora, a potenciar la autonomía de los alumnos, la toma de decisiones conjunta, resolución de problemas, participación proporcional, etc.

Dentro de los métodos didácticos que abordan la didáctica de la traducción jurídica encontramos acercamientos basados en el género textual, en concreto en las dimensiones comunicativa y formal de los géneros de especialidad. El trabajo por géneros textuales permite prestar atención a aquellos aspectos lingüísticos, textuales y estilísticos que la comunidad de práctica en cuestión sanciona como válidos de cara a poder encontrar la solución entre un conjunto de estrategias y posibles soluciones, a priori todas válidas, relacionadas con las características macroestructurales del textos (estructura, secciones) y aspectos intratextuales de los mismos (grado de formalidad, modalidad, conectores, léxico, densidad terminológica y fraseológica). Al fin y al cabo, géneros textuales "makes it possible to identify a series of elements, such as the agents playing the roles of sender and receiver, the relationship that is established between them in terms of power or authority, the degree of specialisation they offer and the situational context in which the genre in question occurs" (Montalt et alii, 2008).

Por su parte, Garzone (2007: 211-216) y Pontrandolfo (2016) proponen un método basado en la aproximación gradual a la complejidad de los textos jurídicos que explore la diversificación vertical del discurso jurídico. Pontrandolfo (ibid.: 53) señala que es precisamente la especificidad y la diversificación vertical del discurso jurídico la que puede potenciar el uso de materiales auténticos en el aula desde el inicio del aprendizaje de la traducción jurídica en tanto en cuanto se haga una selección cuidada de los materiales del curso. La aproximación vertical, continúa (ibid.: 54) puede permitir a los docentes de traducción jurídica centrarse, al menos en los estadios iniciales de la educación de sus alumnos, en problemas más básicos sin tener que profundizar en aspectos culturales relacionados con el derecho comparado, por ejemplo. 
Prieto (2011: 14) presenta un método para la traducción jurídica entendida como proceso basado en una secuencia de cuatro pasos: análisis del skopos y macro-contextualización del texto original, partiendo del ordenamiento jurídico en que se insierte un texto, la rama del derecho a la que pertenece y la tipología textual en concreto; análisis del texto origen, animando a los alumnos a recurrir a técnicas de razonamiento jurídico y hermenéutica jurídica para solucionar problemas de ambigüedad (ibid.: 15); transferencia y producción del texto meta, donde se atajan los problemas relacionados con el anisomorfismo entre los ordenamientos jurídicos implicados a través de técnicas de derecho comparado que permitan a los alumnos dirimir el grado de equivalencia entre conceptos jurídicos; y revisión textual, donde se evalúa la adecuación del texto producido en relación a su skopos.

Por último, Way (2016: 1019) sugiere un modelo basado en el análisis crítico del discurso apuntalado por un marco operativo de toma de decisiones (ibid., 2014) capaz de activar las sub-competencias que conforman la competencia traductora jurídica. Al igual que Prieto arriba, el método de Way, que recurre a un modelo pedagógico centrado en el alumno a través de una metodología de trabajo por proyectos y tareas, se centra en la traducción como proceso, de manera que los alumnos pueden evaluar las dificultades que encuentran en el proceso de traducción y así desarrollar la confianza necesaria en su habilidad de toma de decisiones (ibid.: 143). El método que propone Way va más allá de la toma de decisiones específica para un problema de traducción en particular de un texto concreto, sino que busca promover un marco operativo general que beba de las diferentes sub-competencias del alumnado y les permita movilizar estrategias clave en el proceso de traducción. Tal y como afirma la autora (2016: 1021): "By locating the text within the discursive practice (production, distribution, consumption), students become familiar with the internalised social structures and conventions governing the text", lo que les permite identificar las prácticas discursivas y sociales similares y divergentes entre las culturas implicadas como punto de inicio para la traducción del texto.

\section{La metodología en el aula de traducción jurídica según sus guías docentes}

En un intento de indagar acerca de los métodos y estrategias metodológicas que utilizan los docentes de traducción jurídica en España y poder 
compararlos con los modelos teóricos de competencia traductora y los modelos de aula explorados en este artículo, nos propusimos analizar el conjunto de guías docentes de las materias de traducción jurídica que se ofertan en España en los planes de estudio de grado. El análisis de guías docentes debería proporcionar información de primera mano acerca de aquellas cuestiones metodológicas sobre las que nos preguntamos en el presente artículo, por lo que, en un primer estadio de la investigación, analizamos los planes de estudio de todos los grados de traducción ofertados en España y extrajimos 94 guías docentes correspondientes a asignaturas de traducción jurídica.

A simple vista, se constató que un número importante de guías docentes carecía de un apartado de "metodología" propiamente dicho, el apartado estaba en blanco, o la información que contenía estaba más relacionada con otros elementos de la guía (contenido, evaluación) que con las estrategias metodológicas que se movilizan en el aula. Otro elemento problemático que apareció con cierta frecuencia en el estudio de las guías docentes fue que, en general, la sección de metodología en las guías de las asignaturas de una misma universidad eran sospechosamente similares, incluso a veces idénticas, independientemente del docente responsable, el número de alumnos previsto o el curso académico en que se imparte. Si bien esto podría deberse a esfuerzos titánicos de coordinación docente por parte de los responsables de estas asignaturas, surgen dudas razonables acerca de la utilidad de la guía docente como reflejo de la realidad en el aula, lo que restaría valor a la misma guía docente en tanto que contrato entre los alumnos que se matriculan en una asignatura y el docente que la imparte.

Pese a las limitaciones encontradas, se aprecia una cierta tendencia a vincular las asignaturas de traducción jurídica con modelos pedagógicos mixtos centrados ligeramente en la figura del docente como gestor de la materia. Así, la sección de metodología suele hacer referencia explícita al docente como agente activo mediante referencias directas ("el docente presentará los conceptos teóricos que se estudiarán en el aula"; "los profesores responsables presentarán el marco teórico relacionado con los contenidos del programa") o mediante el uso de pasivas reflejas ("se espera que los alumnos revisen los contenidos teóricos antes de llegar al aula"; "se facilitará a los alumnos una lista de materiales de lectura obligatoria"). El concepto de clase magistral aparece en todas las guías docentes analizadas, sea mediante referencia directa (Universitat 
d'Alacant, Universidad de Córdoba) o mediante el calificativo "clase magistral colaborativa" (Universitat Jaume I, Universitat d'Alacant) donde los docentes buscan la participación del alumnado durante la explicación y presentación de contenidos. La clase magistral se justifica sistemáticamente por el peso conceptual de la traducción jurídica.

Igualmente, las guías hacen referencia a principios de aprendizaje colaborativo y otros elementos post-positivistas de la educación como contrapunto a las sesiones teóricas, sea mediante referencia directa (Universidad Europea, Universidad de Las Palmas, Universidad de Valladolid, Universidad de Granada, Universidad Pablo de Olavide, etc.) o por referencia indirecta a través de las diferentes estrategias y recursos que movilizan: debates y presentaciones orales de los alumnos (Universidad Alfonso X el Sabio, Universidad Complutense de Madrid, Universidad de Alcalá, etc.) o evaluación mediante portfolios, cuestionarios de autoevaluación o evaluación entre pares (Universidad de Valladolid, Universidad de Murcia, etc.).

Las guías docentes tienden a resaltar el papel protagonista de las tareas y proyectos de traducción, sean individuales o en grupo, aunque parece que la mayor parte de docentes optan por pedir a sus alumnos que traduzcan en casa y corregir posteriormente en el aula, comentando aquellos aspectos problemáticos y dificultades que han encontrado. Muy pocas guías docentes mencionan la autenticidad de los textos (Universitat Jaume I, Universidad de Las Palmas, Universidad de Murcia, Universidad Pablo de Olavide), un elemento al que la literatura parece otorgar especial importancia.

Además de la práctica de la traducción se mencionan actividades de pre-traducción tales como la identificación de problemas de traducción, propuestas de traducción para unidades discursivas inferiores al texto, ejercicios de comprensión de discursos jurídicos, búsqueda de textos paralelos, corrección de traducciones, evaluación crítica de traducciones, traducciones comentadas, estudios de caso, creación de glosarios, presentaciones orales, etc.

Por último, respecto a la socialización de los alumnos en sus respectivas comunidades de práctica, la Universitat Pompeu Fabra, por ejemplo, habla de la reflexión acerca de aquellos aspectos profesionales asociados a la profesión del traductor; la Universitat Jaume I presenta a sus alumnos encargos que reproducen las condiciones de trabajo de traductores jurídicos en activo, subrayando cuestiones de facturación 
o tarifas de traducción; y la Universidad Alfonso X el Sabio menciona cuestiones relacionadas con planes de negocios para traductores y gestión de proyectos de traducción.

Como puede apreciarse, el breve análisis de las guías docentes que presentamos dibuja un panorama de la enseñanza de la traducción jurídica en España donde el peso del campo conceptual marca de forma pronunciada el método pedagógico y la metodología empleada: por una parte, el docente suele cobrar un papel central en lo que se refiere a la organización y gestión del curso y los contenidos teóricos. Por otra, las sesiones prácticas de traducción tienden a adoptar una metodología más variada, de corte constructivista.

\section{La metodología en el aula de traducción jurídica según sus docentes}

Como contrapunto al análisis de las guías docentes de los módulos de traducción jurídica se llevaron a cabo una serie de entrevistas semiestructuradas entre los propios docentes responsables de las asignaturas. Dado el reducido tamaño de la población objeto de estudio, apenas un centenar de docentes en toda España, se aplicaron métodos de muestreo no probabilísticos basados en los siguientes criterios de exclusión:

- Docentes a tiempo completo en universidades españolas con docencia en módulos de traducción jurídica.

- Docentes con, al menos, quince años de experiencia en educación superior, de cara a contar con su capacidad de evaluar la evolución en la didáctica de la traducción jurídica.

- Mayor representatividad geográfica posible, es decir, no más de un docente de traducción jurídica de la misma universidad.

- Disponibilidad para entrevista síncrona, para dotar de fluidez a las entrevistas y poder encontrar vías y enfoques diferentes no previstos a priori por el entrevistador.

En total, se grabaron y trascribieron siete entrevistas, de alrededor de una hora de duración cada una de ellas. Las entrevistas cubrieron temas relacionados con la metodología en el aula y métodos pedagógicos de los docentes de traducción jurídica, el trabajo por competencias y el estado actual de la enseñanza de la traducción jurídica en España. 
Respecto a la metodología docente, todos los entrevistados se refirieron a su aula como un espacio donde confluye la teoría y la práctica aunque, por razones económicas y de gestión del grado, la mayoría de ellos se vean obligados a separar taxativamente las sesiones teóricas de las prácticas, con grupos de diferentes tamaños y aulas diferentes (con y sin ordenadores). Solo dos de los entrevistados tenían grupos del mismo tamaño independientemente del tipo de sesión y podían combinar ambas indistintamente, siempre en aula con ordenadores. En ese sentido, un argumento utilizado por la gran mayoría de los entrevistados (seis de los siete) fue el del número de alumnos como elemento limitante a la hora de introducir algún cambio en su metodología o plantearse algún enfoque diferente. El séptimo, voz discordante, argumentó que si bien es cierto que tener muchos alumnos en el aula hace más difícil la corrección individual o grupal de tareas, no hay obligación alguna por parte del profesorado de hacer tal seguimiento, sino que se trata de una obligación autoimpuesta. La obligación no es la de corregir, apuntó, "sino asegurarse de que los alumnos, al final del semestre, pueden identificar problemas de traducción, solucionarlos, encontrar las herramientas y recursos necesarios y evaluar la calidad de una traducción".

En relación con el tipo de actividades que se realizan en el aula y fuera de ella, no hubo una opinión mayoritaria. Dos de los entrevistados, dadas las pocas horas de contacto que tienen con los alumnos, se decantaron por la opción de traducir en casa y debatir opciones de traducción en el aula; mientras que, para otros dos, traducir en clase es imprescindible para poder andamiar el proceso de aprendizaje de los alumnos, ayudándolos precisamente cuando más se necesita la ayuda: al traducir. La disponibilidad y tipo de aula fue otro de los elementos limitantes a la hora de diseñar las actividades, ya que en muchos casos los docentes no contaban con aulas equipadas con ordenadores. En tales casos, uno de los entrevistados afirmó lo siguiente: "lo que haces es trabajar otros aspectos, como la toma de decisiones. Trabajas más el cómo llegar a una solución de un problema de traducción a si la solución en sí es adecuada o no. Los textos con los que trabajas en el aula son un pretexto para que los alumnos puedan sistematizar procesos de traducción".

Además de la práctica de la traducción, los docentes mencionaron la necesidad de introducir actividades y tareas de complejidad creciente, desde el análisis y la caracterización de textos y géneros jurídicos a 
encargos complejos de traducción, pasando por la revisión y corrección de traducciones, presentaciones orales, o creación de glosarios específicos por bloques temáticos. El contenido conceptual fue, asimismo, objeto de debate entre los entrevistados. Un argumento utilizado frecuentemente es la aparente falta de interés por parte de los alumnos en el aprendizaje del contenido declarativo de la asignatura en detrimento del saber operativo, procedimental, cosa que perjudica su proceso de aprendizaje. En palabras de un entrevistado: “(...) Ellos quieren hacer. Hacer cosas. Estudiar el contenido no es tan relevante por la supuesta competencia instrumental y de documentación que ellos dicen que tienen. Y que desde luego no tienen". Otro argumento esgrimido fue el dominio de las lenguas de trabajo, tanto la lengua origen como la lengua meta. El sentir general de los entrevistados es que los alumnos no cuentan, en ninguna de sus dos lenguas, con habilidades de comprensión y expresión que les permitan acceder a textos jurídicos o producirlos, con lo que en el aula muchas veces se debe dedicar tiempo a cuestiones que no están estrictamente relacionadas con la traducción jurídica y que deberían haberse trabajado previamente en el currículum.

En ese sentido, con la reforma de los planes de estudio y la armonización del sistema universitario español en el EEES, los entrevistados mencionaron la dificultad creciente de impartir contenidos de traducción jurídica en un plan de estudios que, al tener menos créditos que las antiguas licenciaturas, ha prescindido, en un número significativo de casos, de antiguas asignaturas instrumentales de contenido teórico que preparaban a los alumnos para la traducción jurídica (Introducción al Derecho, Inglés jurídico, etc.). Tal y como mencionaron más de la mitad de los entrevistados, estas asignaturas permitían a los alumnos familiarizarse con el campo de especialización de forma progresiva desde un número de perspectivas diferentes, un buen punto de inicio para introducirlos en la traducción jurídica.

\section{Discusión y conclusiones}

En este artículo hemos presentado un primer esbozo del panorama actual en la enseñanza de la traducción jurídica en España. Para ello, hemos contextualizado, situado, la didáctica de la traducción jurídica en el seno del EEES y los modelos pedagógicos que promueve, para después vincularlo a la necesidad de socializar a nuestros alumnos en 
sus respectivas comunidades de práctica. Sin caer en la trampa de la mercantilización de la educación superior (Biesta, 2013) hemos recalcado la necesidad de crear entornos de aprendizaje capaces de conjugar la formación de agentes interlingüísticos que conozcan la norma consensuada por la profesión pero que puedan, asimismo, evaluarla críticamente; sin olvidar las amenazas y los cambios a los que se enfrenta la profesión a corto y medio plazo, en particular aquellos relacionados con la tecnología y la automatización de procesos.

En paralelo, hemos relacionado los modelos de aula contemporáneos con el paradigma de la formación por competencias. Dada su naturaleza teórico-práctica-actitudinal, el trabajo por competencias parece vehicular satisfactoriamente los principios pedagógicos que promueve el EEES. En el caso concreto de la didáctica de la traducción jurídica, hemos subrayado el peso del campo conceptual como elemento clave en la conceptualización de modelos de competencia traductora, tal y como hemos contemplado en la revisión teórica de modelos de competencia específicos.

Por último, en el caso de la metodología en el aula, hemos llevado a cabo una triple triangulación de datos: por una parte, a través de aquellos métodos docentes y estrategias metodológicas que la literatura considera esenciales en la educación contemporánea; por otro, el estudio de las guías docentes de los módulos de traducción jurídica en España y la propia experiencia docente de aquellos responsables de la educación en traducción jurídica de sus alumnos. Como hemos visto, el análisis empírico arroja como resultado modelos de aula eclécticos que beben de tradiciones pedagógicas diferentes, algunas de ellas más centradas en el docente, sobre todo en lo que se refiere al contenido teórico de las asignaturas de traducción jurídica, y algunas de ellas en línea con los principios del trabajo colaborativo en entornos que imitan la realidad socioprofesional.

Si bien la educación superior parte con la aparente desventaja de tener un cuerpo docente predominantemente lego en cuestiones pedagógicas más allá de su propia experiencia e intuición, en los últimos años el interés por la didáctica de la traducción, y en particular el de la traducción jurídica, parece haber dado pie a un debate fundamentado sobre las razones que apuntalan nuestra praxis docente con miras a mejorar la educación de nuestros alumnos. De este intercambio dialógico de experiencias, ideas y bases pedagógicas dependerá, mayormente, 
nuestra habilidad de crear entornos de aprendizaje específicos y efectivos en los que los futuros egresados de los planes de estudio de traducción puedan adquirir las destrezas específicas que les permita pasar de una acción periférica legítima al centro mismo de sus comunidades de práctica.

\section{Referencias}

Andújar Moreno, Gemma \& Cañada Pujols, Maria Dolores. 2011. El enfoque por tareas en la didáctica de la traducción jurídica: propuesta de aplicación. Estudios de Traducción 1: 185-204. doi: http://doi.org/10.5209/ rev

Ausubel, David P. 1960. The use of advance organizers in the learning and retention of meaningful verbal material. Journal of Educational Psychology 51: 267-272.

Biel, Łucja. 2011. Professional Realism in the Legal Translation Classroom: Translation Competence and Translator Competence. Meta: Journal Des Traducteurs 56(1): 162-178. doi: http://doi.org/10.7202/1003515ar

Biesta, Gert. 2013. Interrupting the Politics of Learning. Power and Education, 5(1), 4. http://doi.org/10.2304/power.2013.5.1.4

Borja Albi, Anabel. 2007. Estrategias, materiales y recursos para la traducción jurídica. Castelló: Edelsa/Edicions de la Universitat Jaume I.

Borja Albi, Anabel. 2013. A genre analysis approach to the study of the translation of court documents. Linguistica Antverpiensia, New Series Themes in Translation Studies (LANS - TTS) 12: 33-53.

Borja Albi, Anabel. \& Martínez-Carrasco, Robert. 2019. Future-proofing legal translation: a paradigm shift for an exponential era. In Simonnæ, Ingrid \& Kristiansen, Maritta (eds.) Legal Translation: Current Issues and Challenges in Research, Methods and Applications. Berlín: Frank \& Timme, 187-206.

Cao, Deborah. 2007. Translating Law. Clevedon \& Buffalo \& Toronto: Multilingual Matters.

Cao, Deborah. 2014. Teaching and learning legal translation. Semiotica 201: 103-119. doi: http://doi.org/10.1515/sem-2014-0022

Casacuberta Nolla, Francisco \& Peris Abril, Álvaro. 2017. Traducción automática neuronal. Revista Tradumàtica. Tecnologies de La Traducció 15(15): 66-74. doi: http://doi.org/10.5565/rev/tradumatica.203

Chesterman, Andrew. 1997. Memes of translation: the spread of ideas in translation theory. Ámsterdam/Filadelfia: John Benjamins.

De Bot, Kess, Lowie, Wander \& Verspoor, Marjolijn. 2007. A Dynamic Systems Theory approach to second language acquisition. Bilingualism: 
Language and Cognition 10(1): 7-21. doi: http://doi.org/10.1017/ S1366728906002732

Edwards-Schachter, Mónica, García-Granero, Ana, Sánchez-Barrioluengo, Mabel, Quesada-Pineda, Henry \& Amara, Nabil. 2015. Disentangling competences: Interrelationships on creativity, innovation and entrepreneurship. Thinking Skills and Creativity 16: 27-39. doi: http://doi. org/10.1016/j.tsc.2014.11.006

EHEA Ministerial Conference. 2015. Yerevan Communiqué.

EHEA Ministerial Conference. 2018. Paris communiqué.

EMT Expert Group. 2009. Competences for Professional Translators, Experts in Multilingual and Multimedia Communication.

Galán Mañas, Anabel. 2011. Translating authentic technical documents in specialised translation classes. JoSTrans 16: 109-125.

Garzone, Giuliana. 2007. Osservazioni sulla didattica della traduzione giuridica. In Mazzota, P. \& Salmon, L. (eds.) Tradurre le microlingue scientifico-professionali. Riflessioni teoriche e proposte didattiche. Torino: UTET, 194-238.

González-Davies, María \& Enríquez-Raído, Vanessa. 2016. Situated learning in translator and interpreter training: bridging research and good practice. The Interpreter and Translator Trainer 10(1): 1-11. doi: http://doi. org/10.1080/1750399X.2016.1154339

González-Davies, María \& Scott-Tennet, Christopher. 2005. A problem-solving and student-centred approach to the translation of cultural references. Meta: Journal Des Traducteurs 50(1): 160-179.

Göpferich, Susanne. 2013. Translation competence. Explaining development and stagnation from a dynamic systems perspective. Target 25(1): 61-76.

Halász, Gabor \& Michel, Alain. 2011. Key competences in Europe: Interpretation, policy formulation and implementation. European Journal of Education 46(3): 289-306. doi: http://doi.org/10.1111/j.14653435.2011.01491.x

Hansen, Gyde. 1997. Success in Translation. Perspectives: Studies in Translatology 5(2): 201-210.

Hertog, Erik. 2001. Aequitas: access to justice across language and culture in the EU. Antwerpen: Lessius Hogeschool.

Hertog, Erik. 2003. Aequalitas: equal access to justice across language and culture in the EU. Antwerpen: Lessius Hogeschool.

Hertog, Erik. 2009. Reflection forum on multilingualism and interpreter training: final report. Bruselas: EC.

Kelly, Dorothy. 2002. Un modelo de competencia traductora: bases para el diseño curricular. Puentes 1: 9-20. doi: http://doi.org/10.1007/s13398014-0173-7.2 
Kelly, Dorothy. 2008. Training the Trainers: Towards a Description of Translator Trainer Competence and Training Needs Analysis. TTR: Traduction, Terminologie, Rédaction 21(1): 99-125. doi: http://doi. org/10.7202/029688ar

Kiraly, Donald. 2000. A Social constructivist approach to translator education : empowerment from theory to practice. Manchester, UK: St. Jerome.

Kiraly, Donald. 2016. Authentic Project Work and Pedagogical Epistemologies: A Question of Competing or Complementary Worldviews? In Kiraly, D. (ed.) Towards Authentic Experiential Learning in Translator Education. Mainz: V\&R Press, 53-66.

Kordić, Ljubica. 2016. Developing Translation Competence Within the Lifelong Learning Programme for Lawyer-Linguists in the Republic of Croatia. Studies in Logic, Grammar and Rhetoric 45(1): 97-110. doi: http://doi.org/10.1515/slgr-2016-0018

Lasnier, François. 2001. Un modèle intégré pour l'apprentissage d'une compétence. Pédagogie Collégiale 15(1): 28-33.

Lave, Jean \& Wenger, Etienne. 1991. Situated Learning: Legitimate Peripheral Participation. Cambridge: Cambridge University Press.

Li, Defeng F. 2001. Language teaching in translator training. Babel 47(4): 343-354.

Lommel, Arle \& DePalma, Donald A. 2017. Language Pair Demand and Supply: 2017.

Lowe, Pardee. 1987. Revising the ACTFL/ETS Scales for a New Purpose: Rating Skill in Translating. In Translation Excellence: Assessment, Achievement, Maintenance. New York: SUNY Binghamton Press, 53-61.

Macías Otón, Elena. 2016. Aproximación didáctica a la enseñanza- aprendizaje de la traducción de terminología y fraseología jurídicas. Quaderns. Revista de Traducció 23: 133-154.

Marco, Josep. 2016. On the margins of the profession: the work placement as a site for the literary translator trainee's legitimate peripheral participation. The Interpreter and Translator Trainer 0417(agosto). doi: http:// doi.org/10.1080/1750399X.2016.1154341

Martínez-Carrasco, Robert. 2017a. El aprendizaje de la traducción en el seno del EEES: una justificación de la metodología de aula. Revista Iberoamericana de Lingüistica 12: 161-180.

Martínez-Carrasco, Robert. 2017b. Epistemological Approaches to Legal Translation Education: A Situated Account. Universitat Jaume I.

Montalt Ressurrecció, Vicent, Ezpeleta Piorno, Pilar \& García Izquierdo, Isabel 2008. The acquisition of translation competence through textual genre. Translation Journal 12(4). 
Neubert, Albrecht. 2000. Competence in Language, in Languages and in Translation Developing. In Schäffner, Christina \& Adab, Beverly (eds.) Translation Competence . Ámsterdam: John Benjamins, 3-18.

Nord, Christiane. 1991. Text Analysis in Translation. Theory, Methodology, and Didactic Application of a Model for Translation - Oriented Text Analysis. Amsterdam/Atlanta: Rodopi.

Ordóñez-López, Pilar. 2015. A critical account of the concept of "basic legal knowledge": Theory and practice. Interpreter and Translator Trainer 9(2): 156-172. doi: http://doi.org/10.1080/1750399X.2015.1051768

PACTE. 2003. Building a Translation Competence Model. In Alves, F. (ed.) Perspectives in Process Oriented Research. Ámsterdam: John Benjamins.

Piecychna, Beata. 2013. Legal translation competence in the light of translational hermeneutics. Studies in Logic, Grammar and Rhetoric 34(47): 141-159. doi: http://doi.org/10.2478/slgr-2013-0027

Pontrandolfo, Gianluca. 2016. Aproximación gradual a la traducción jurídica: un recorrido didáctico. The Journal of Specialised Translation 26: 50-71.

Prieto Ramos, Fernando. 2011. Developing Legal Translation Competence: An Integrative Process-Oriented Approach. Comparative Legilinguistics International Journal for Legal Communication 5: 7-21.

Pym, Anthony. 1991. A definition of translational competence applied to the teaching of translation. In Jovanivic, M. (ed.) Proceedings of the 12th World Congress of FIT "Translation: A Creative Profession". Belgrade: Prevodilac, 541-546.

Šarčević, Susan. 1997. New Approach to Legal Translation. The Hague/London: Kluwer Law International.

Scarpa, Federica \& Orlando, Daniele 2017. What it takes to do it right: an integrative EMT-based model for legal translation competence. JoSTrans. The Journal of Specialised Translation 27: 483-487.

Schäffner, Christina \& Adab, Beverly. (2000). Developing translation competence. Ámsterdam: John Benjamins.

Soriano-Barabino, Guadalupe. (2018). La formación del traductor jurídico: análisis de la competencia traductora en traducción jurídica y propuesta de programa formativo. Quaderns. Revista de Traducción 25, 217-229.

Stansfield, Charles, Scott, Mary \& Kenyon, Dorry. (1992). The Measurement of Translation Ability. The Modern Language Journal 76(4): 455-467.

Toury, Gideon. 1995. Descriptive Translation Studies and Beyond. Ámsterdam: John Benjamins.

Way, Catherine. 2014. Structuring a Legal Translation Course: A Framework for Decision-Making in Legal Translator Training. In Cheng, Le, Sin, Kin Kui \& Wagner, Anne (eds.) The Ashgate Handbook of Legal 
Translation. Law, Language and Communication. Farnham: Ashgate Publishing Company.

Way, Cathrine. 2016. The Challenges and Opportunities of Legal Translation and Translator Training in the 21st Century. International Journal of Communication 10: 1009-1029.

Wilss, Wolfram. 1989. Towards a Multi-facet Concept of Translation Behavior. Target 1(2): 129-149.

World Economic Forum. 2017. The Global Competitiveness Report. Geneva. 